\title{
Single-Step Application of Polyelectrolyte Complex Films as Oxygen Barrier Coatings
}

\author{
Jiaying Li, Gerard van Ewijk, Derk Jan van Dijken, Jasper van der Gucht,* and Wiebe M. de Vos* \\ Cite This: ACS Appl. Mater. Interfaces 2021, 13, 21844-21853 \\ Read Online
}

ABSTRACT: Polyelectrolyte complex (PEC) films such as polyelectrolyte multilayers have demonstrated excellent oxygen barrier properties, but unfortunately, the established layer-by-layer approaches are laborious and difficult to scale up. Here, we demonstrate a novel single-step approach to produce a PEC film, based on the use of a volatile base. Ammonia was used to adjust the $\mathrm{pH}$ of poly(acrylic acid) (PAA) so that direct complexation was avoided when it was mixed with polyethylenimine (PEI). Different charge ratios of homogeneous PEI/PAA solutions were successfully

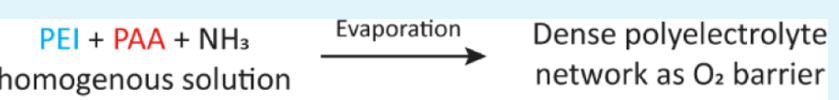
prepared and phase diagrams varying the concentration of
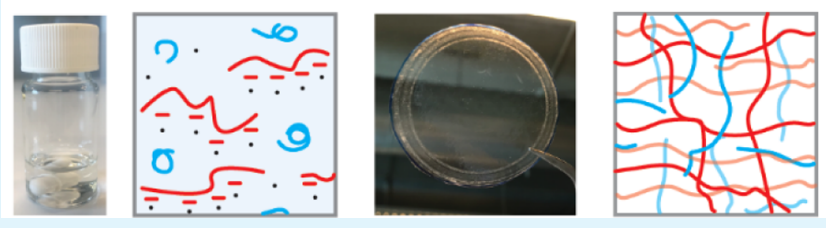
ammonia or polyelectrolyte were made to study the phase behavior of PEI, PAA, and ammonia in water. Transparent $\sim 1 \mu \mathrm{m}$ thick films were successfully deposited on biaxially orientated polypropylene (BOPP) sheets using a Meyer rod. After casting the films, the decrease in $\mathrm{pH}$, caused by the evaporation of ammonia, triggered the complexation during drying. The oxygen permeation properties of films with different ratios and single polyelectrolytes were tested. All films displayed excellent oxygen barrier properties, with an oxygen permeation below $4 \mathrm{~cm}^{3} \cdot \mathrm{m}^{-2} \cdot \mathrm{day}^{-1} \cdot \mathrm{atm}^{-1}(<0.002$ barrer$)$ at the optimum ratio of $2: 1 \mathrm{PEI} / \mathrm{PAA}$. This ammonia evaporation-induced complexation approach creates a new pathway to prepare PEC films in one simple step while allowing the possibility of recycling.

KEYWORDS: polyelectrolyte complexation, phase diagrams, waterborne coatings, gas barrier, food packaging

\section{INTRODUCTION}

Thin flexible films with good barrier properties are important for food preservation. Commonly used food packaging materials are laminated multilayers with metal oxides as the gas barrier layer; however, metalized packaging generally lacks transparency, flexibility, and recyclability. ${ }^{1}$ To improve these properties, scientists have been focusing mostly on developing polymer matrices with impenetrable nanocomponents, meanwhile balancing the mechanical, optical, and barrier properties. The choices of polymers can be the common synthetic polymers such as polyethylene and polypropylene or bio-based polymers like poly(lactic acid). ${ }^{2}$ The choices of the nanocomponents are more diverse, ranging from metal oxides $\left(\mathrm{TiO}_{2}, \mathrm{ZnO}\right)^{3,4}$ and two-dimensional (2D) sheets (graphene oxide, layered double hydroxide, and nanoclays $)^{5-7}$ to biobased fillers (cellulose nanomaterials). ${ }^{8} \mathrm{~A}$ recent review has summarized the possible composite materials and their processing routes, where the final reduction in oxygen permeation can vary from as low as $5.6 \%$ to almost $100 \%$.

Among these promising approaches, one frequently mentioned method is the use of polyelectrolytes (PEs). PEs are a special class of charged polymers that are usually watersoluble. When oppositely charged PEs meet, they tend to complex into different forms. ${ }^{10}$ PEs are often used to build films using a layer-by-layer (LbL) approach, where a thin transparent polyelectrolyte multilayer (PEM) can be deposited on a charged substrate by repeatedly dip-coating the substrate into cationic and anionic PE solutions. ${ }^{11}$ PEM-based films with added nanocomponents, such as nanoclays or graphene oxide, have demonstrated excellent gas barrier properties that lead to a $>99 \%$ reduction in oxygen permeation.,12-14 However, multicomponent films remain a substantial challenge for recycling. Without adding other components, PE-only films have also been constructed using LbL, and it was observed that the dense ionically cross-linked network also lowered the gas permeation significantly. ${ }^{15-18}$ The LbL approach is a straightforward method and has nanometer-level control over the film structure; however, the extensive film deposition and washing steps limit the possibility to scale up and industrialize.

A two-step film deposition method was developed by Haile et al. in 2017. ${ }^{19}$ Using two weak polyelectrolytes: polyethylenimine (PEI) and poly(acrylic acid) (PAA), $\mathrm{pH}$ and salt concentration were tuned to form liquid coacervates with

Received: March 17, 2021

Accepted: April 20, 2021

Published: April 29, 2021 
processible viscosities. The formed coacervates were then cast using a Meyer rod, after which stronger complexation and solidification were induced by immersion into a buffer bath. Compared with the LbL approach, this method is much more simplified. However, the composition and concentration of the coacervates remained unknown. In a more recent study, Smith et al. proposed a method to form a solution of poly(diallyldimethylammonium chloride) (PDDA) and PAA. By adjusting the $\mathrm{pH}$ to 2, PAA remained uncharged and could thus be homogeneously mixed with PDDA. After the film formation by dip-coating, again a bath was used to induce complexation. $^{20}$ The advantage of this solution-based approach is that the PE concentration and ratio could be controlled. For both methods, the aqueous immersion step provides the environment for introducing the necessary $\mathrm{pH}$ switch. It is a controlled procedure in which different parameters can be tuned, such as acid/base types, $\mathrm{pH}$ value, salt types, and their concentrations. However, the thickness is limited as once a dense top layer is formed, the lower part takes too long to be further complexed. Moreover, since PE chains may become mobile in the aqueous environment, it is difficult to detect whether the composition of the films remains the same as that of the initial preparation. For instance, excess polymer chains and salt ions can transfer from the coating into the bath.

In this work, we report a unique and simple one-step approach to the formation of a polyelectrolyte complex (PEC) film, with excellent control over the final film thickness and composition. PAA and PEI under normal $\mathrm{pH}$ conditions would complex in solution. However, by adding a volatile base, PEI becomes uncharged and can be mixed with PAA to form a homogeneous solution. Here, we use ammonia $\left(\mathrm{NH}_{3}\right)$, which is already commonly used in the paint and coating industries. High-concentration solutions with various ratios of PEI, PAA, and $\mathrm{NH}_{3}$ were prepared to study their phase behaviors. Here, we use branched PEI instead of linear PEI because commercially available linear PEI has low molecular weights, which is not suitable for the film formation, and because branched PEI has been well studied in the LbL system for various applications. ${ }^{21,22}$ Selected solutions were then cast using a Meyer rod onto biaxially orientated polypropylene (BOPP) substrates. During $\mathrm{NH}_{3}$ evaporation, the $\mathrm{pH}$ reduction allows the PEs to complex, leading to films that demonstrate excellent oxygen barrier properties as shown in Scheme 1. This method of controlling $\mathrm{pH}$ has several advantages over both the LbL approach and the aqueous bath approach. Films with different thicknesses can be prepared in a simple manner, while the top dense layer may slow down or stop the complexation in an aqueous bath. Moreover, coating various substrates is possible, including paper. Above all, the layer composition is well controlled beforehand, as all nonvolatile components become part of the complex layer. The final advantage of this one-step method is that by exposing the film to $\mathrm{NH}_{3}$, the films can be dissolved again. This reversibility makes recycling possible while the films can maintain their properties under normal conditions.

\section{EXPERIMENTAL SECTION}

2.1. Materials. Branched polyethylenimine (PEI, average $M_{\mathrm{w}}$ $25000 \mathrm{~g} \cdot \mathrm{mol}^{-1}, \leq 1 \%$ water), poly(acrylic acid) (PAA, average $M_{\mathrm{w}}$ $100000 \mathrm{~g} \cdot \mathrm{mol}^{-1}, 35 \mathrm{wt} \%$ in water), ammonia $\left(\mathrm{NH}_{3}\right.$, for analysis EMSURE ISO, Reag. Ph Eur, $25 \%$ in water), hydrochloric acid ( $\mathrm{HCl}$, ACS reagent, 37\%), sodium hydroxide $(\mathrm{NaOH},>98 \%$ pellets $)$, sodium chloride $(\mathrm{NaCl}, \mathrm{ACS}$ reagent, $\geq 99.0 \%)$, methyl red (ACS
Scheme 1. Schematic Illustration of $\mathrm{NH}_{3}$ EvaporationInduced Complexation $^{a}$

a.

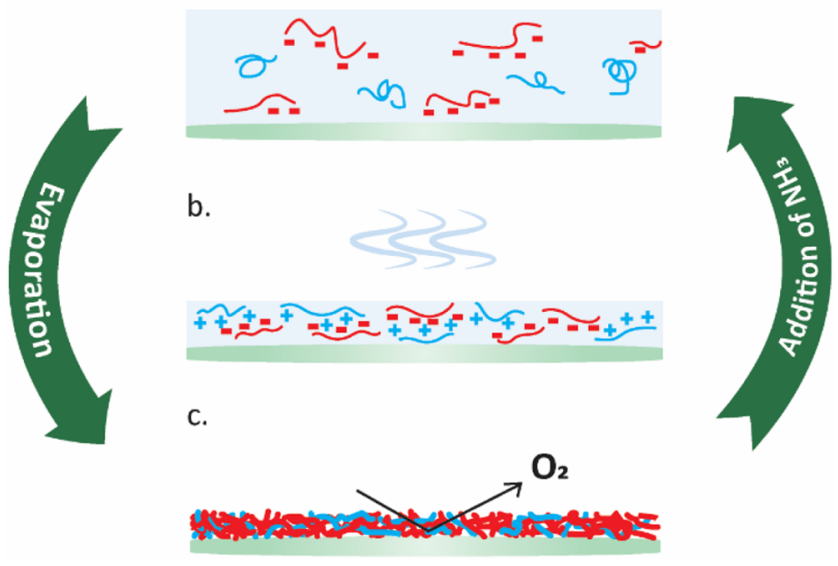

(a) PEI (blue) is kept uncharged within the mixture by adding $\mathrm{NH}_{3}$ into PAA (red) solution. (b) The solution is then cast onto a substrate. During drying, $\mathrm{NH}_{3}$ evaporation lowers the $\mathrm{pH}$ to the point where complexation starts. (c) A dense ionic cross-linked film that provides oxygen barrier properties is formed after all solvent has evaporated. This process is reversible by adding aqueous $\mathrm{NH}_{3}$ to the dried film, allowing the recyclability of the PEs and the plastic support.

reagent, crystalline), and thymol blue (ACS reagent) were all purchased from Sigma-Aldrich (the Netherlands). All water used was deionized water (Milli- $\mathrm{Q}$, Merck, the Netherlands). All chemicals were used as received. Biaxially orientated polypropylene (BOPP) sheets were provided by BASF and purchased from Hapece B.V., the Netherlands.

2.2. Preparation of Phase Diagrams. 2.2.1. Varying $\mathrm{NH}_{3}$ Concentration. PEI was diluted to $20 \mathrm{wt} \%$ using deionized water. PAA 35 wt \% stock solution was first diluted with $\mathrm{NH}_{3}$ solution to 30 , 25 , and $20 \mathrm{wt} \%$. Then, these solutions were further diluted to $20 \mathrm{wt}$ $\%$ with deionized water. With this control, there were samples with four levels of $\mathrm{NH}_{3}$ concentrations including a blank. Different ratios of PEI/PAA were prepared from 4:1 to $1: 3$. The mixing ratio was based on the charge ratio such that every primary, secondary, and tertiary amine group of PEI was considered as a potential charge site, while PAA has one charge site per monomer $\left(M_{\mathrm{EI}}=43.04 \mathrm{~g} \cdot \mathrm{mol}^{-1}\right.$ and $M_{\mathrm{AA}}$ $\left.=72.07 \mathrm{~g} \cdot \mathrm{mol}^{-1}\right)$.

2.2.2. Varying PE Concentration. PEI $100 \mathrm{wt} \%$ stock was diluted with water to $35,20,10$, and 5 wt $\%$. PAA 35 wt $\%$ stock solution was also diluted with water to 20,10 , and 5 wt $\%$. Four sets $(35,20,10,5$ wt \%) with different ratios of PEI/PAA mixtures were prepared following the same charge ratio.

2.2.3. Adjusting $\mathrm{pH}$ before Mixing. Three sets of different $\mathrm{pH}(5$, $7,9)$ samples were prepared. The $\mathrm{pH}$ of $20 \mathrm{wt} \%$ PAA and PEI were first adjusted to the same $\mathrm{pH}$ value 5,7 , or 9 by adding $5 \mathrm{~mol} \mathrm{HCl}$ or $\mathrm{NaOH}$. After reaching the desired $\mathrm{pH}$, they were diluted to $10 \mathrm{wt} \%$ PE. Different ratios of mixtures were prepared.

All of the samples were stirred vigorously with a stirring bar for 15 min after which different phases of materials (homogeneous solution, coacervate, complex) were obtained. Photos of these phase diagram samples were all taken when the phases were stable $(>48 \mathrm{~h})$. Depending on the appearance of the mixtures, they were identified as a complex (solid in an aqueous phase), a coacervate (a liquid PE rich phase coexisting with a dilute phase), or a solution (one transparent phase). Then, the $\mathrm{pH}$ of each sample was measured using $\mathrm{pH}$ strips ( $\mathrm{pH}$ 0-14 Universal indicator, Merck, the Netherlands). To obtain more accurate $\mathrm{pH}$ results, the eyedropper tool from Adobe Illustrator was used to extract colors for comparisons (Figure S1).

To visually track the $\mathrm{pH}$ changes, $\mathrm{pH}$ indicators were added into PEI/PAA 1:1 (thymol blue) and 1:3 (methyl red). For comparison, a 
1:1 sample was also prepared with $\mathrm{NaOH}$. Samples were then brushed using a painting brush onto paper to better observe the color changes. The drying processes were recorded, and photos of these samples were taken when they were just applied and after they were completely dried ( $\sim 30 \mathrm{~min})$.

2.3. Preparation of Casting Solutions. For the film casting, the solutions with the maximum amount of $\mathrm{NH}_{3}$ (PAA diluted with $\mathrm{NH}_{3}$ from 35 to $20 \mathrm{wt} \%$ ) were used. For each ratio, to ensure PAA is neutralized and the final $\mathrm{pH}$ is basic, excess $\mathrm{NH}_{3}$ was used. The amount of $\mathrm{NH}_{3}$ thus increased with the increase of PAA. The total PE concentration was kept at $20 \mathrm{wt} \%$ with changing ratios. The compositions at each ratio are shown below (Table 1). The PAA

Table 1. Composition and Calculated Density of PEI/PAA at Different Ratios

$\begin{array}{cccccc}\begin{array}{c}\text { charge ratios } \\ \text { PEI/PAA }\end{array} & \begin{array}{c}\text { PEI } \\ \text { (wt \%) }\end{array} & \begin{array}{c}\mathrm{PAA} \\ (\text { wt \%) }\end{array} & \begin{array}{c}\mathrm{NH}_{3} \\ (\text { wt \%) }\end{array} & \begin{array}{c}\mathrm{H}_{2} \mathrm{O} \\ (\text { wt \%) }\end{array} & \begin{array}{c}\text { calculated density of } \\ \text { dried films }\left(\mathrm{g} \cdot \mathrm{mL}^{-1}\right)\end{array} \\ 4: 1 & 14.1 & 5.9 & 3.2 & 76.8 & 1.10 \\ 3: 1 & 12.8 & 7.2 & 3.8 & 76.2 & 1.12 \\ 2: 1 & 10.9 & 9.1 & 4.9 & 75.1 & 1.15 \\ 1: 1 & 7.5 & 12.5 & 6.7 & 73.3 & 1.22 \\ 1: 2 & 4.6 & 15.4 & 8.3 & 71.7 & 1.28 \\ 1: 3 & 3.3 & 16.7 & 8.9 & 71.1 & 1.31\end{array}$

solution was added to the PEI solution at once. In total, $4 \mathrm{~g}$ of solution mixture was prepared for each ratio. Each solution was stirred vigorously with a stirring bar for $30 \mathrm{~min}$ and was judged ready for use when all bubbles had disappeared.

2.4. Film Fabrication and Thickness Control. BOPP substrates were cleaned with water and ethanol, then pretreated with an oxygen plasma (Femto plasma cleaner, Diener electronic GmbH, Ebhausen, Germany) to introduce hydrophilicity. To examine the control over thickness, PE films at a ratio of 1:1 were cast using a casting machine (BYK Instrument) with 10, 25, 50, and $100 \mu \mathrm{m}$ Meyer rods. Triplicate samples for each thickness were prepared and all films were dried inside the fume hood. Another method to examine the overall film thickness was by calculation, which was done to avoid damaging the film before gas permeation measurements. The thickness of BOPP substrates was first measured using a micrometer. For each substrate, the thickness was measured at 10 randomly chosen locations and the average result was calculated. To control the surface area of all films, bare BOPP was cut into $5 \mathrm{~cm} \times 16 \mathrm{~cm}$ strips for casting. After drying, the coated samples were cut into $5 \mathrm{~cm} \times 6 \mathrm{~cm}$ strips then weighed. The densities of $100 \%$ PEI $\left(1.03 \mathrm{~g} \cdot \mathrm{mL}^{-1}\right.$ at $25^{\circ} \mathrm{C}$ provided by SigmaAldrich) and PAA (1.40 g. $\mathrm{mL}^{-1}$ calculated from $35 \mathrm{wt} \% 1.14 \mathrm{~g} \cdot \mathrm{mL}^{-1}$ at $25{ }^{\circ} \mathrm{C}$, provided by Sigma-Aldrich) were used to estimate the density at the different ratios of PEI/PAA. The density of each ratio is listed in Table 1. The total thickness of the film was then calculated using eq 1

$$
d_{\text {total }}(\mu \mathrm{m})=d_{\mathrm{BOPP}}+\left(\frac{\Delta m}{\rho \cdot s}\right) \times 10^{4}
$$

where $d_{\mathrm{BOPP}}(\mu \mathrm{m})$ is the average thickness measured by a micrometer, $\Delta m(\mathrm{~g})$ is the weight difference between after and before coating the film, $\rho\left(\mathrm{g} \cdot \mathrm{mL}^{-1}\right)$ is the calculated density of the film, and $s\left(\mathrm{~cm}^{2}\right)$ is the surface area of the film, which was $5 \mathrm{~cm} \times 6 \mathrm{~cm}$ for all films. After the gas permeation measurements, the total thickness of each film was measured by a micrometer to compare with $d_{\text {total }}$.

For further oxygen permeation measurements, films with single PE (PEI or PAA) and with the mixtures at the different ratios were prepared using the wet $10 \mu \mathrm{m}$ rod.

2.5. Film Characterizations. Fourier transform infrared (FT-IR) spectroscopy (Spectrum Two, PerkinElmer) was used to examine pure PEs (PAA, PAA with ammonia, PEI) and the mixed films. Freestanding pieces of PE films were prepared on a hydrophobic Teflon plate, followed by drying in the fume hood. To remove water, all samples were dried under vacuum at $30{ }^{\circ} \mathrm{C}$ for $24 \mathrm{~h}$. The measurements were conducted in the reflectance mode at a spectral resolution of $4 \mathrm{~cm}^{-1}$ from wavenumber 400 to $4000 \mathrm{~cm}^{-1}$. For each measurement, a minimum of 16 scans was conducted.

Film morphology and defects were examined by a scanning electron microscope (SEM, JSM-6010LA, JEOL, Japan). All samples were stored under vacuum at $30{ }^{\circ} \mathrm{C}$ for $24 \mathrm{~h}$ before imaging. To induce conductivity, a $\mathrm{Pt} / \mathrm{Pd} 5 \mathrm{~nm}$ coating was sputtered on the samples (Quorum Q150T ES, Quorum Technologies, Ltd., U.K.).

To show the film responses to water, dried freestanding pieces of PE films were put into deionized water and stirred for $5 \mathrm{~min}$ with a stirring bar. A 3:1 PEI/PAA oxygen permeation test sample was put into water and then ammonia to demonstrate the possibility of recycling.

2.6. Oxygen Permeation Test. The oxygen permeation of the films was measured by a gas separation setup. Samples were prepared on the previous day and stored in the fume hood. All measurements were performed at the same conditions at $30{ }^{\circ} \mathrm{C}$ and $0 \%$ relative humidity. The applied pressure was 3 bar. Before starting measurements, films were degassed under vacuum for $6 \mathrm{~h}$. Each sample was measured for $48 \mathrm{~h}$. The permeability is defined as barrer, shown in eq 2

$$
\text { permeability }(\text { barrer })=3.35 \times 10^{-16} \frac{n \cdot d_{\text {total }}}{s \cdot t \cdot P}
$$

where $n(\mathrm{~mol})$ is the amount of oxygen collected, $d_{\text {total }}(\mathrm{m})$ is the thickness of the film, $s\left(\mathrm{~m}^{2}\right)$ is the surface area of the sample, $t(\mathrm{~s})$ is the time used for collecting $n \mathrm{~mol}$ of oxygen, and $P$ is the pressure difference.

The amount of gas collected in a certain volume was controlled by setting the end pressure, based on the ideal gas law (eq 3)

$$
n(\mathrm{~mol})=\frac{\left(P_{\text {end }}-P_{\text {start }}\right) V}{R T}
$$

where $P_{\text {end }}$ was set to be 2 mbar; $P_{\text {start }}$ should be around 0 (under vacuum), then the unit of pressure was converted to $\mathrm{Pa} ; V\left(\mathrm{~m}^{3}\right)$ is the cell volume, $R$ is the ideal gas constant $8.3145 \mathrm{~m}^{3} \cdot \mathrm{Pa} \cdot \mathrm{K}^{-1} \cdot \mathrm{mol}^{-1}$, and $T(\mathrm{~K})$ is the temperature $(303.15 \mathrm{~K})$.

For the final permeation data, the system leakage was considered and excluded from the raw data. The leakage rate was around $2 \times$ $10^{-5} \mathrm{mbar} \cdot \mathrm{s}^{-1}$, with small variations among four different cells. Samples of bare BOPP sheets, single PEI, single PAA, and mixed films at six different ratios were measured. For each data point, at least three samples were measured, and the average results with standard deviations were reported. The permeation data in barrer were also converted into OTR $\left(\mathrm{cm}^{3} \cdot \mathrm{m}^{-2} \cdot \mathrm{day}^{-1} \cdot \mathrm{atm}^{-1}\right)$ for comparisons (eqs S1 and S2).

\section{RESULTS AND DISCUSSION}

3.1. Phase Diagrams. To characterize PE complexation, its phase behavior was studied. PE mixtures can exist as a solution, coacervate, or solid complex depending on various parameters, such as $\mathrm{pH}, \mathrm{PE}$ concentration, mixing ratio, and salt concentration. ${ }^{23}$ For our application, the transition from a homogeneous solution to a solid complex is the key. Here, we use high-concentration solutions instead of coacervates for several reasons. Coacervates are not homogeneous right after mixing and it can require days or even weeks for them to stabilize. The formed coacervates have high viscosities that make casting difficult and they cannot be diluted with water to control the viscosity. Adding water to coacervates again leads to phase separation. Most importantly, the composition and the concentration of the coacervate remain unknown. Thus, we focus on solutions that can be homogeneously mixed with a controlled ratio. To form a continuous film, rather than separated particles, the concentration should be high to ensure a continuous network throughout the drying process. 
Many phase diagrams in the literature have proven that the PE phase can be tuned from a solution to a solid complex by varying $\mathrm{PE}$ concentration, $\mathrm{PE}$ molecular weight, $\mathrm{PE}$ mixing ratio, salt concentration, and using miscible solvents. ${ }^{24-29}$ Surprisingly, there is no thorough study of $\mathrm{pH}$-induced phase transitions, even though $\mathrm{pH}$ is one of the most important parameters for tuning weak polyelectrolytes. The complexity of adjusting $\mathrm{pH}$ is that the salt concentration would usually also change. Sun et al. were the first to demonstrate a successful phase transition of PEI and PAA by tuning $\mathrm{pH}^{30}$ In our chosen system, both PEI and PAA were used in their uncharged forms without other counter-ions, while the $\mathrm{pH}$ was tuned by $\mathrm{NH}_{3}$, which gave us a system only containing ammonium ions $\left(\mathrm{NH}_{4}^{+}\right)$. This is important because in our one-step film deposition method, introducing salt ions may lead to salt crystallization/inhomogeneity during drying. An example of possible defects is shown in Figure S2. Thus, phase diagrams with varying $\mathrm{NH}_{3}$ concentration and $\mathrm{PE}$ concentration were constructed.

First, the effect of the $\mathrm{NH}_{3}$ concentration for mixtures prepared at different ratios was studied, since $\mathrm{pH}$ is a crucial parameter that influences the degree of ionization of both PEI and PAA. Pictures of the samples at different $\mathrm{pH}$ and mixing ratios are shown in Figure S3. Figure 1a shows that a homogeneous solution phase can be achieved for each ratio when sufficient $\mathrm{NH}_{3}$ (no higher than $5 \mathrm{wt} \%$ ) is added. As the $\mathrm{NH}_{3}$ concentration decreases, there is a phase transition from solution to coacervate (PEI/PAA ratios 4:1-2:1) or solution directly to solid complex (ratios 1:1-1:3). For the film formation, the solutions with the highest concentration of $\mathrm{NH}_{3}$ were used. As $\mathrm{NH}_{3}$ evaporates, the final film composition would be expected to follow the same transitions. This indicates that most ratios can reach the dense complex state that is desired for gas barrier properties.

The $\mathrm{pH}$ of the different samples (in Figure $1 \mathrm{~b}$, the same data are replotted) was obtained using $\mathrm{pH}$ indicator paper because a $\mathrm{pH}$ electrode could not be used due to the high viscosity of the samples. Since the smallest $\mathrm{pH}$ difference that can be detected with these universal $\mathrm{pH}$ paper strips is approximately one $\mathrm{pH}$ unit, the accuracy is limited. For example, both solution and coacervate phases were found for a polymer ratio 4:1 at pH 11 . Probably, the $\mathrm{pH}$ change required for this transition is too small to be detected. PEI and PAA are both weak polyelectrolytes so that $\mathrm{pH}$ directly influences their degree of ionization. PAA has a $\mathrm{p} K_{\mathrm{a}}$ value around $4.5^{31}$ and when the $\mathrm{pH}$ is around 2 it is not charged, while at a $\mathrm{pH}$ reaching 10 it becomes fully charged. ${ }^{32}$ The branched PEI has three different $\mathrm{p} K_{\mathrm{a}}$ values due to its primary (4.5), secondary (6.7), and tertiary (11.6) amines. ${ }^{33}$ Oppositely to PAA, PEI is fully charged at low $\mathrm{pHs}(<4)$ and almost uncharged at high $\mathrm{pHs}$ $(>10) .{ }^{34}$

In general, our phase diagram shows that the complexation is successfully avoided by keeping PEI almost uncharged at high $\mathrm{pH}$ around $10-11$, while complexation occurs at lower $\mathrm{pH}$, where both PEI and PAA are charged. There is also a clear effect of the mixing ratio, with high PEI/PAA ratios (4:1-2:1) complexing at substantially higher $\mathrm{pH}$ values than the low PEI/ PAA ratios (1:1-1:3). Most likely PEI remains partially charged even at a $\mathrm{pH}$ of 10 , still allowing macroscopic phase separation when there is a sufficient excess of PEI. However, when PAA is in excess, the $\mathrm{pH}$ has to be sufficiently low for PEI to induce macroscopic phase separation. Otherwise, small negatively charged soluble complexes may be formed with one (a)
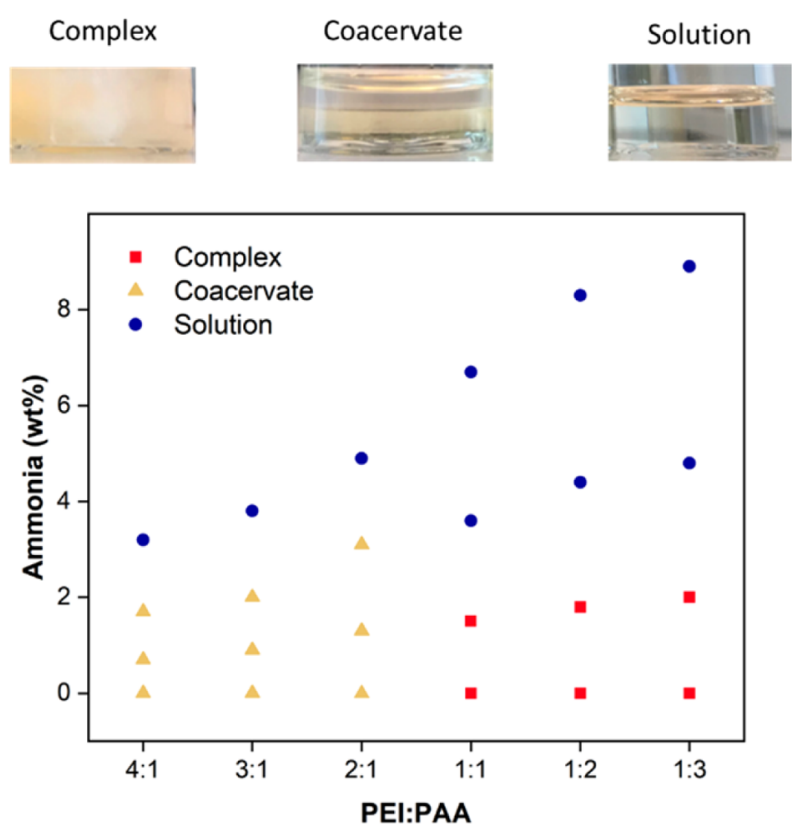

(b)

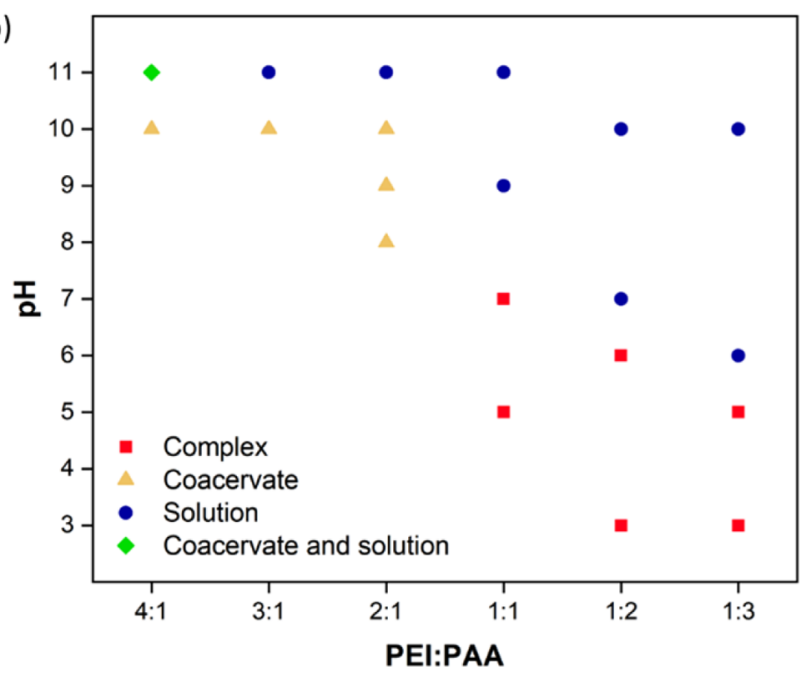

Figure 1. (a) Phase diagram of PEI/PAA at different ratios (based on the number of chargeable groups) with varying $\mathrm{NH}_{3}$ concentration. The final mixture can exist as a solid complex, liquid coacervate, or homogeneous solution. (b) Same phase diagram shown as a function of $\mathrm{pH}$.

PEI chain interacting with a few PAA chains present in the solution.

Next, the effect of PE concentration on the phase behavior was studied, to determine whether the evaporation of water would also be able to induce a phase transition. Pictures of the samples and measured $\mathrm{pH}$ values are shown in Figure S4. The $\mathrm{PE}$ concentration range studied starts at $5 \mathrm{wt} \%$ and ends at 35 wt $\%$, due to viscosity limitations. Within this concentration range of $5-35 \%$ wt $\%$, no phase transition was observed. As shown in Figure 2a, samples at PEI/PAA ratios 4:1-2:1 all formed coacervates while from 1:1 to 1:3 they formed solid complexes. This indicates that water evaporation alone cannot trigger a phase transition when concentrating PEs from 5 to 35 wt \%. It is noticeable that for excess PEI ratios, complexes were first formed; then, they turned into a coacervate. For example, the PE phase was between a complex and a coacervate for ratio 
(a)

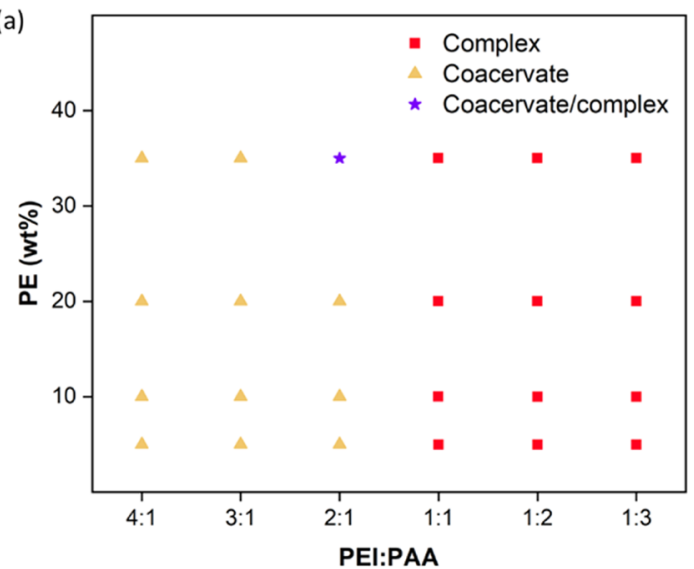

(b)

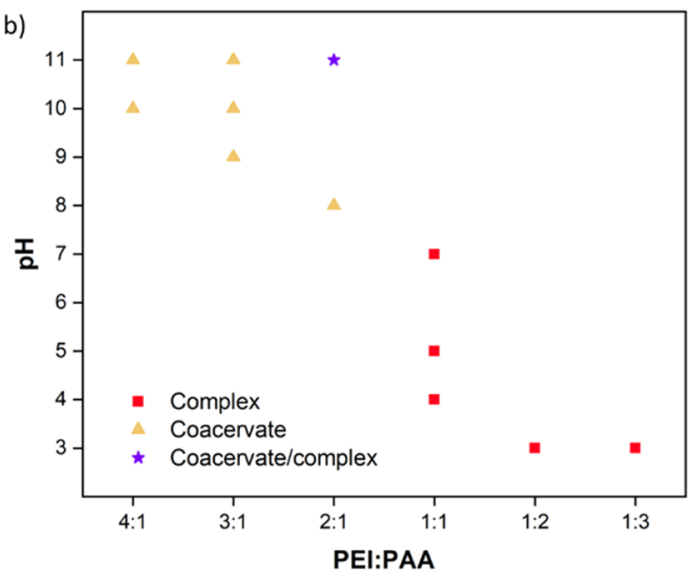

Figure 2. (a) Phase diagram of PEI/PAA at different PEI/PAA ratios upon varying $\mathrm{PE}$ concentration. The final mixture can exist as a solid complex or coacervate. (b) $\mathrm{pH}$ value of each sample.

2:1 at 35 wt \%. Our data do not exclude the occurrence of phase transitions when the PE concentration exceeds $35 \mathrm{wt} \%$. It is possible that when the $\mathrm{PE}$ concentration exceeds a certain value during drying, the chains may be "quenched" in the complex form due to a loss of mobility.

The $\mathrm{pH}$ values of the various samples are also replotted in Figure $2 \mathrm{~b}$ to connect the $\mathrm{pH}$ value to the obtained phase. Interestingly, even at $\mathrm{pH} 11$, the ratios $4: 1,3: 1$, and $2: 1$ were able to form a coacervate or complex instead of a solution at high polymer concentration. One explanation is the limited accuracy of $\mathrm{pH}$ measurements. Another is that the additional $\mathrm{NH}_{4}{ }^{+}$helps to avoid complexation by charge screening. Apart from that, these data are consistent with the first phase diagram (Figure 1), showing that the mixtures with excess PEI form coacervates, while the ratios with excess PAA form solid complexes at sufficiently low $\mathrm{pH}$.

To study the complexation, while eliminating the effect of $\mathrm{pH}$ change, PEI and PAA solutions were adjusted to have the same $\mathrm{pH}(5,7,9)$ before mixing. Within this phase diagram (Figure 3), the effect of added $\mathrm{HCl}$ or $\mathrm{NaOH}$ on the salt concentration was neglected, as the $\mathrm{pH}$ is expected to have a stronger influence compared to the small changes in salt concentration that stem from setting the $\mathrm{pH}$. These three $\mathrm{pH}$ values were chosen because they correspond to cases where PEI and PAA are almost equally charged $(\mathrm{pH}=7)$, where PEI is more charged than PAA $(\mathrm{pH}=5)$, and where PEI is less charged than PAA $(\mathrm{pH}=9)$. The pictures of the samples and the $\mathrm{pH}$ of the mixtures are included in Figure S5. Comparing

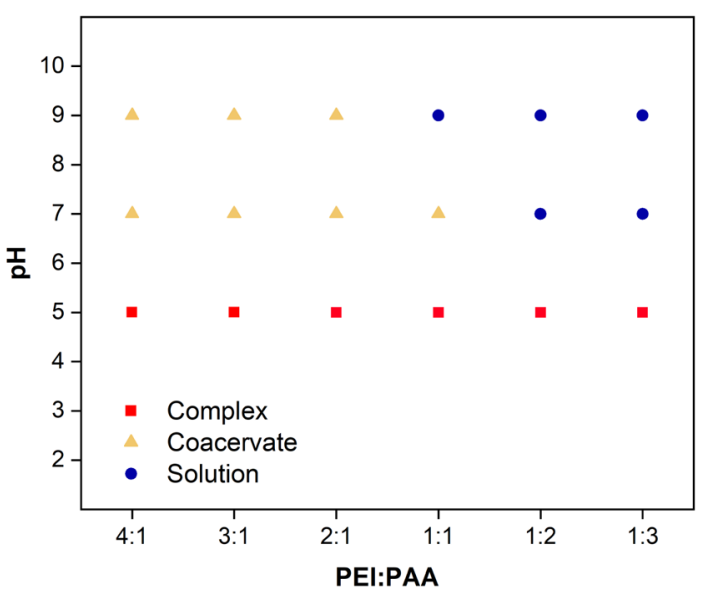

Figure 3. Phase diagram of PEI/PAA at different PEI/PAA ratios when adjusting PEI and PAA solutions to the desired $\mathrm{pH}(5,7,9)$.

samples with the same $\mathrm{pH}$, coacervates were formed when PEI was in excess for $\mathrm{pH}=7$ and 9, while for $\mathrm{pH}=5$, solid complexes were formed at all ratios, probably because of the higher charge density of PEI. Using the ratio 1:1 as an example, an acidic environment is required again to ensure that PEI is sufficiently charged. One difference compared to the previous observations is that at a ratio of $1: 1$ at $\mathrm{pH} 7$, a solid complex was formed in previous phase diagrams while here it is a coacervate.

Summarizing all three phase diagrams, it can be concluded that excess charged PEI is required to form a solid complex. This can be achieved in two ways, either by increasing the PEI concentration at fixed $\mathrm{pH}$ or by decreasing the $\mathrm{pH}$ at fixed PEI concentration. This result is in agreement with the study of Sun et al., who found that solid precipitates ("nonfluidity state") formed when the $\mathrm{pH}$ was low enough ( 4) or when PEI was in excess. They also proposed a structural rearrangement of the preliminary complex into a precipitated complex, either a turbid colloidal suspension or one phase soluble complex depending on the PEI/PAA ratio and $\mathrm{pH}^{30}$ The difference with our study is that we used PEI and PAA with much higher $M_{w}$, which enhances the macroscopic phase separation. Combining with our $\mathrm{pH}$ data, the possible structural changes are summarized in Figure 4. Due to the branched nature of PEI and the difference in $M_{w}$, the PAA chain acts as the long backbone, while PEI as more compact coils. ${ }^{27}$ For a fixed ratio, PEI gets less charged and PAA gets more charged when $\mathrm{pH}$ increases. The solid complex then acquires a net negative charge, which weakens the interaction and leads to a liquid-like coacervate and eventually to soluble complexes. Eventually, when the $\mathrm{pH}$ is too high, the complexes fall apart because the electrostatic attraction vanishes when PEI becomes uncharged. For a fixed $\mathrm{pH}$, the ratios with more PEI are more likely to form a precipitate. The circled samples in Figure 4 showed more than one phase behavior at the same $\mathrm{pH}$; one possible reason can be the limited accuracy in the $\mathrm{pH}$ measurements. Another reason can be the difference in salt concentrations. At $\mathrm{pH} \mathrm{11}$, the samples from phase diagram 1 appeared as solutions due to the presence of $\mathrm{NH}_{4}^{+}$, while the samples from phase diagram 2 (zero salt ions) appeared as coacervates. A similar case occurred for the samples at $\mathrm{pH} 7$ that the sample from phase diagram 3 (containing $\mathrm{Na}^{+}$and $\mathrm{Cl}^{-}$) appeared as coacervate, while the other two samples from phase diagrams 1 and 2 appeared as complex. 


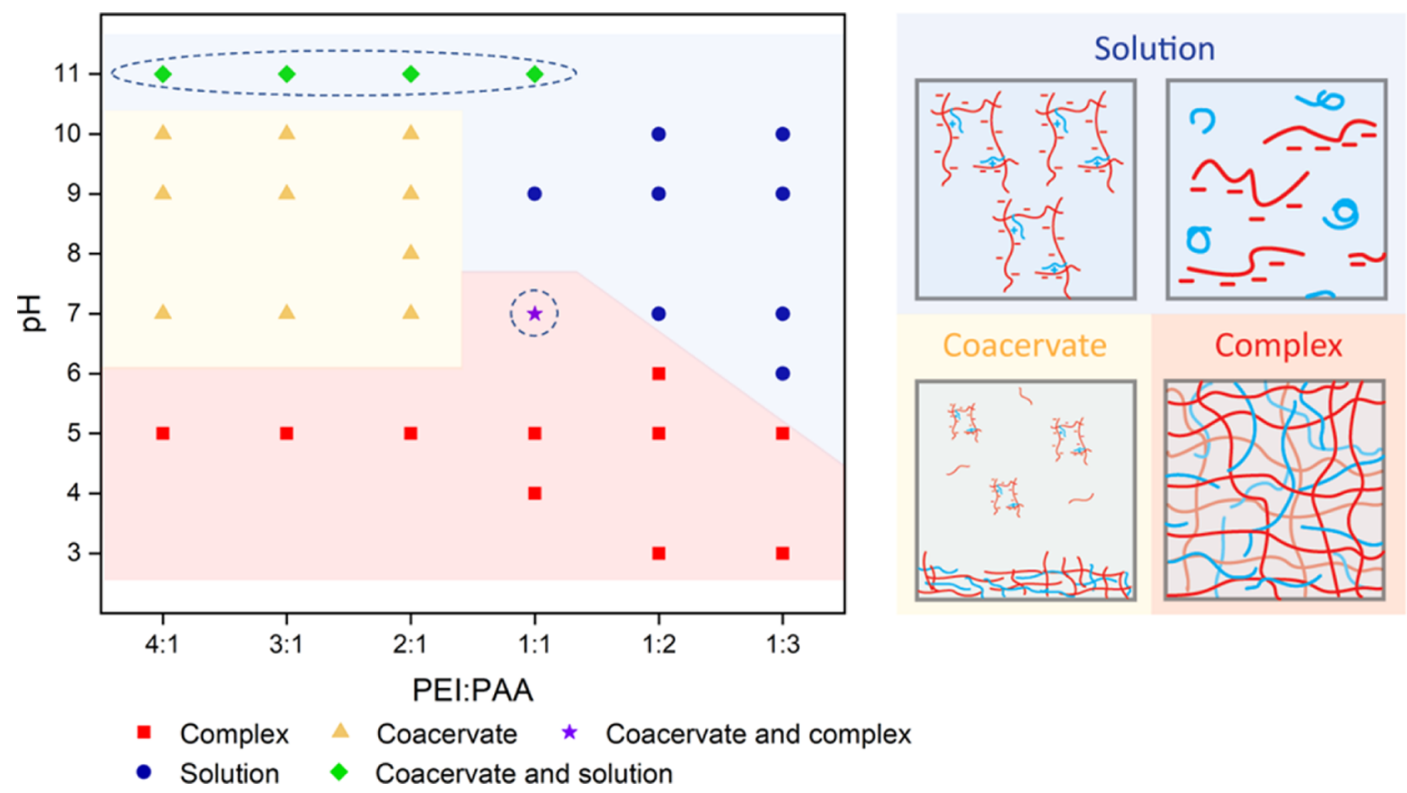

Figure 4. Possible PEC structures according to $\mathrm{pH}$ and ratio changes.

Translating the information obtained from the phase diagrams to the proposed drying process, it can be expected that the phase transitions during drying are dominated by $\mathrm{NH}_{3}$ evaporation and the resulting decrease of the $\mathrm{pH}$. Although water evaporation happens simultaneously, it only concentrates the PEs without inducing phase transitions in the studied range 5-35 wt \%. However, the loss of mobility at close-to-dry high concentrations may also contribute to complex formation. Once $\mathrm{NH}_{3}$ has completely evaporated, the $\mathrm{pH}$ returns to the original $\mathrm{pH}$. This starting $\mathrm{pH}$ was predetermined by the mixing ratio. The $\mathrm{pH}$ at every ratio was therefore different due to the acidity of PAA and the basicity of PEI. This explains why 4:1 and 3:1 ratios, with excess PEI, can only form coacervate because excess PEI also increases the $\mathrm{pH}$. To monitor whether the expected decrease of the $\mathrm{pH}$ occurs during drying, $\mathrm{pH}$ indicators were used to track the $\mathrm{pH}$ change during the drying process (Figure S6). For PEI/PAA ratios $1: 1$ and 1:3, the $\mathrm{pH}$ required for solid complexation was indeed reached. By contrast, when the nonvolatile base $\mathrm{NaOH}$ was used to adjust the $\mathrm{pH}$, the $\mathrm{pH}$ did not decrease. The videos of the drying processes are also available in the Supporting Information (Videos S1 and S2).

3.2. Film Preparation and Characterization. To show control over the thickness of the films, PE films at a ratio of 1:1 were prepared using different Meyer rods (wet 10, 25, 50, and $100 \mu \mathrm{m}$ thick). Figure 5 shows that the dried film thickness increases linearly with increasing Meyer rod thickness. The solutions used contain an overall $20 \mathrm{wt} \% \mathrm{PE}$ and the final dried film thickness should therefore be around one-fifth of the wet thickness. Indeed, the thicknesses measured by a micrometer were similar to these values. However, when comparing the measured film thicknesses with the calculated thickness, assuming the densities reported in Table 1 , the measured thickness is approximately $30 \%$ higher. This indicates that residual water remains trapped in the films, because of the hydrophilic nature of the PEs. To avoid film damage before the permeation test, thicknesses of the samples were estimated by calculation. We postulate that the final thicknesses are close to the calculated ones in these

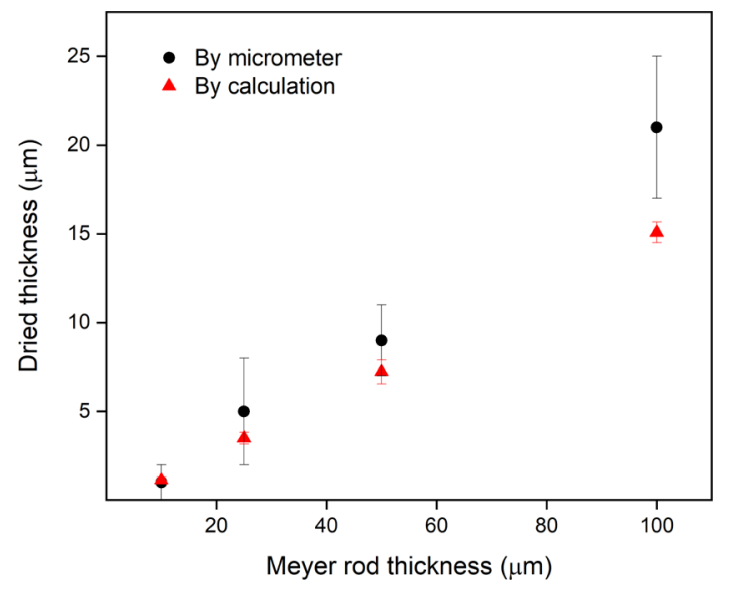

Figure 5. Thickness control with Meyer rods (10, 25, 50, $100 \mu \mathrm{m})$. Final dried thicknesses were measured by a micrometer and compared to the calculated thickness based on the solid content of the solutions.

experiments because these measurements were done at $0 \%$ relative humidity, where residual water will evaporate.

During drying, mechanical stresses can develop in the polymer film due to water loss, which can lead to film damage. This is more likely to occur for thicker films. ${ }^{35}$ Films prepared with a $50 \mu \mathrm{m}$ rod, except those at ratios $4: 1$ and 3:1, all showed cracking while handling, while the top layer of the film prepared at a ratio of 3:1 was delaminated from the BOPP support after the oxygen permeation test. This may result from the measuring condition at $0 \%$ relative humidity and continuous gas flushing. For films prepared with a $25 \mu \mathrm{m}$ rod, the delamination was avoided but microcracks were observed (Figure S7). For the thinnest films, prepared using a $10 \mu \mathrm{m}$ rod, no cracks were observed at any of the PE ratios. Therefore, these films were used for oxygen permeation tests. The development of mechanical stresses was also apparent from the tendency of films to bend after detaching from the substrate. As shown in Figure S8, films prepared with only PEI or PAA and films containing an excess of PEI showed little curling, while films prepared with excess PAA curled up 

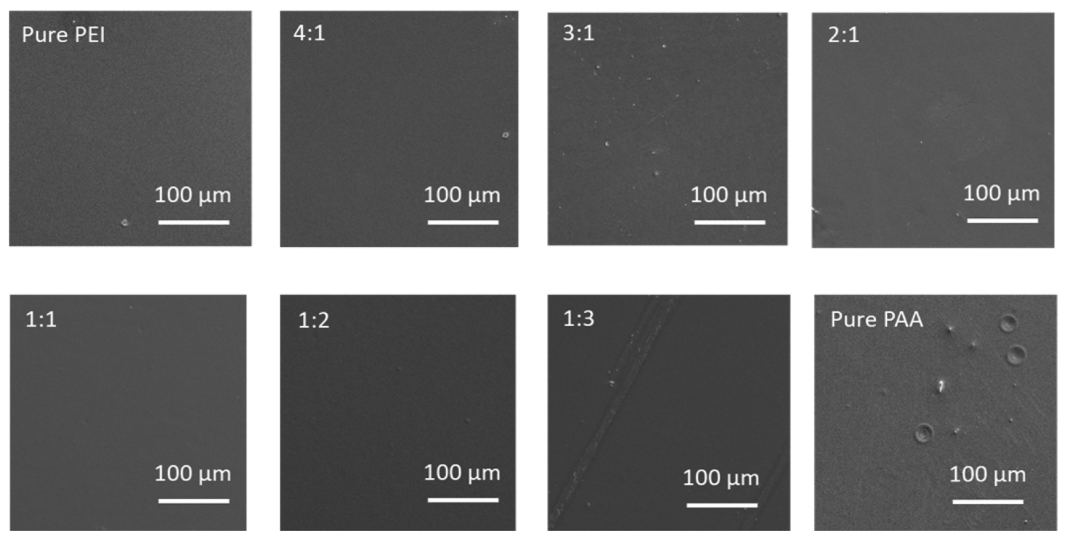

Figure 6. SEM images of films before the oxygen permeation tests to check overall film quality at a low magnification.

significantly, indicating large stresses in these films. This is in agreement with the phase diagram in Figure 1, which shows that films with excess PAA formed solid complexes, which cannot relax mechanical stresses, contrary to films with excess PEI, which stay liquid-like during most of the drying process.

Infrared spectroscopy was utilized to examine the dried PE films. For comparison, single PEs were also measured as dry samples. Pictures of freestanding films of PAA, PEI, and PEI/ PAA at a ratio of 1:1 are shown in Figure S9. PAA is known to absorb ammonia under ambient conditions, and thus some ammonium ions $\mathrm{NH}_{4}{ }^{+}$may remain in the film. ${ }^{36}$ However, FTIR results (see the Supporting Information) cannot prove whether there was residual $\mathrm{NH}_{4}{ }^{+} \cdot{ }^{37}$ In fact, the films are a mixture of PEI and PAA in both charged and uncharged forms so that the exact composition of the films cannot be determined from FT-IR spectra. ${ }^{38,39}$

The transparency of the coated BOPP film is demonstrated in Figure S10. The overall film quality and structure were examined by SEM before the oxygen permeation tests. All PE films dried as a dense layer, and no pores were observed (Figure S11). During the film formation, it was observed that indentations from the wires of the Meyer rod can be generated. As shown in Figure 6, all films appeared to have good film coverage. PAA showed some hole defects that can be caused by dewetting. ${ }^{40}$ For the different $\mathrm{PE}$ ratios, some inhomogeneities could be caused by fast $\mathrm{NH}_{3}$ evaporation. The viscosity of the solution starts to increase immediately due to the onset of the transition to coacervate or complex. This could cause a leveling problem as the indentation from the wires may present. The defects of the BOPP substrate or dust particles can also lead to other small defects like pinholes and cracks (Figure S11). The number of these defects was, however, small and only a limited impact is expected on the overall barrier properties. For the oxygen permeation tests, only the good areas with no visible defects were used. The observations were repeated after the oxygen permeation measurements. No visual cracks of the films were observed, indicating that the layers were stable during the oxygen barrier experiments.

The proposed approach indeed leads to the formation of an optically transparent coating. To demonstrate that the layer consists of PEC instead of dried PEs, some freestanding pieces of the films were obtained and were put into deionized water to examine whether they dissolve or not (Figure S12). For the PEI/PAA ratios 4:1-2:1, the films did not dissolve, clearly indicating complex formation. After stirring, the films became swollen and turned white, similar to previous observations for polyelectrolyte multilayers based on similar PEs; this can be explained by the formation of water pockets that leads to a porous structure. ${ }^{41-43}$ However, for the ratios $1: 1-1: 3$, the films did dissolve in water. The excess of negative charge allows for the formation of soluble complexes, resulting in the removal of the films. Finally, one sample with 3:1 PEI/PAA was first exposed to deionized water, leading to swelling of the coating, after which it was exposed to a 5 wt $\% \mathrm{NH}_{3}$ solution where the film dissolved completely. This reversible complexation process makes it possible to recycle such PE-based films and their plastic supports, which is an advantage over current oxygen barrier coatings.

3.3. Oxygen Barrier Properties. To understand the relationship between film composition and oxygen barrier properties, the oxygen permeation through films consisting of pure PEI or PAA, as well as films with different ratios of PEI:PAA was measured. The permeation of gas through a dense film is mainly dependent on the solubility of the gas molecules in the film and their diffusivity through the film. ${ }^{44}$ When both solubility and diffusivity are minimized, the film should be a good gas barrier. In our case, PAA is more polar than PEI, and as such, the PAA film should have the lowest solubility for nonpolar oxygen. However, the structure of the film also matters and there should be an optimal ratio at which the complexation of the film is maximized to give the densest network structure. The oxygen permeability of bare BOPP substrate was $0.7212 \pm 0.0712$ barrer (OTR $1666 \pm 169 \mathrm{~cm}^{3}$. $\left.\mathrm{m}^{-2} \cdot \mathrm{day}^{-1} \cdot \mathrm{atm}^{-1}\right)$, in line with the literature. ${ }^{45}$ All coated BOPP films showed significantly improved oxygen barrier properties as shown in Figure 7. Surprisingly, pure PEI films gave lower permeation than pure PAA films. A possible explanation is that pure PEI is sticky and that it has the best adhesion with both BOPP and the O-rings that were used for sealing. The drawback is that the prepared PEI film was difficult to handle, and that extra caution was required not to damage the film. On the other hand, pure PAA has the highest permeation and also the largest error. As shown in the SEM images (Figure 6), pure PAA showed dewetting behavior and the coverage of the films may not be as good as for the other films. The relatively poor film quality led to relatively high permeation and poor reproducibility. Interestingly, all PEI/ PAA films showed low permeabilities. There is no clear trend between the permeability and the PEI/PAA ratio, but all films have excellent barrier properties. No significant differences in the permeability could be observed between films with excess PEI (which forms coacervates) and films with excess PAA 


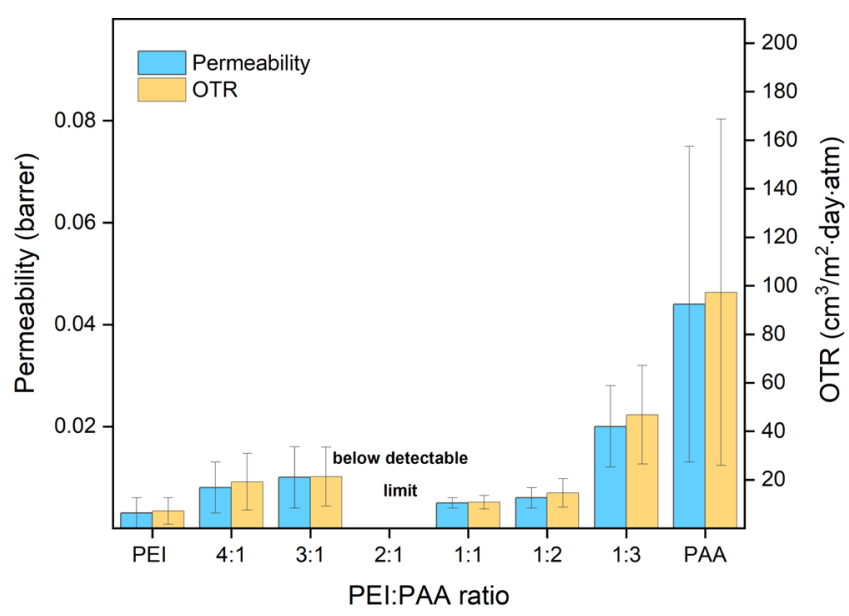

Figure 7. Oxygen permeability and OTR results of single PEs and different ratios.

(which forms solid complexes). The lowest permeability (no permeation could be detected with our setup, $<0.002$ barrer) was found from films with a ratio of $2: 1$. After the measurements, the thicknesses of the samples were measured using a micrometer and the errors were within $1 \mu \mathrm{m}$.

The permeability data were also converted into OTR to compare with the literature that our lowest OTR is below 4 $\mathrm{cm}^{3} \cdot \mathrm{m}^{-2} \cdot$ day $^{-1} \cdot \mathrm{atm}^{-1}$, placing it clearly in the domain of oxygen barrier coatings. According to the literature, it can be used as a gas barrier for products such as oil, snacks, or meat, also depending on the water vapor permeance. ${ }^{46}$ The advantages of this one-step method are: (1) The formed PEC films (ratios 4:1-2:1) are much less water-sensitive compared to pure PE. (2) The ratio of PEI/PAA allows tuning of properties such as gas barrier and mechanical strength. (3) This approach allows the use of paper as a substrate. It is true that our one-step layer did not give as low oxygen permeability as observed in the literature for multistep approaches $(0.013$ for PDDA/PAA, 0.079 for PEI/PAA). ${ }^{19,20}$ Despite the use of completely different measuring methods and a better oxygen barrier substrate PET that they used, one major reason can be the lack of curing steps. In the literature, a low-pH bath and curing after deposition were provided to allow the films to rearrange. Here, a $\mathrm{pH}$ trigger was induced such that the final film $\mathrm{pH}$ was predetermined by the mixing ratio. For ratios $4: 1-2: 1$, the basicity of PEI causes an unfavorable $\mathrm{pH}$ for complexation as PEI is barely charged. This shows that there is certainly potential for our approach to achieving even better barrier properties through further optimization.

\section{CONCLUSIONS}

In this study, we have demonstrated a novel single-step approach to fabricate thin PEI/PAA complex coatings using $\mathrm{NH}_{3}$ evaporation as a trigger. The $\mathrm{pH}$ was first raised to neutralize PAA and uncharge PEI to prepare a homogeneous solution. Then, the $\mathrm{pH}$ decrease by evaporation of $\mathrm{NH}_{3}$ and the charging of PEI by protonation leads to the desired complex formation with PAA. Phase diagrams provided us insight into where the phase transitions start. Homogeneous solutions with known concentrations and ratios were successfully prepared for film formation. Compared to uncoated BOPP, all PE films showed improved oxygen barrier properties. The best film with a PEI/PAA ratio of 2:1 has the lowest permeabilities that they could not be detected by our setup $\left(<0.002\right.$ barrer, OTR $\left.<4 \mathrm{~cm}^{3} \cdot \mathrm{m}^{-2} \cdot \mathrm{day}^{-1} \cdot \mathrm{atm}^{-1}\right)$. The work described here shows that employing volatile bases can provide a completely new route toward PEC films. For the first time, this eliminates the need for tedious and impractical bathing steps and opens the door for real-world, industrial application of these unique materials. On top of this, the excellent oxygen barrier properties make the PEI and PAA complex a promising recyclable barrier material for flexible food packaging. For the following work, we are working on other polyelectrolyte combinations, which may bring better properties and new functionalities. Finally, we see many possibilities to further improve such one-step coatings, for example, with post-treatments. Although the formed films under ambient conditions can be handled without generating cracks or delamination, the mechanical properties of the films are sensitive to any humidity change. Various cross-linking approaches and the addition of plasticizers or nanocomponents can be utilized to further improve the mechanical stability of the films if required. Many directions can be explored to further deepen and exploit this novel approach and eventually on the macroscale.

\section{ASSOCIATED CONTENT}

\section{SI Supporting Information}

The Supporting Information is available free of charge at https://pubs.acs.org/doi/10.1021/acsami.1c05031.

Method to determine $\mathrm{pH}$ values, conversion from barrer to OTR, possible salt crystallization defects, original pictures of phase diagrams and their $\mathrm{pH}$ values, tracking $\mathrm{pH}$ changes with $\mathrm{pH}$ indicators, cracking of thicker films, different curling levels, IR of all PEC and single PEs, possible film defects, transparency of the film, and recyclability of the samples (PDF)

Video of the drying process of a PEI/PAA 1:3 film containing the $\mathrm{pH}$ indicator methyl red (MP4)

Video of the drying process of a PEI/PAA 1:1 film containing the $\mathrm{pH}$ indicator thymol blue (MP4)

\section{AUTHOR INFORMATION}

\section{Corresponding Authors}

Jasper van der Gucht - Physical Chemistry and Soft Matter, Wageningen University and Research, 6708 WE Wageningen, The Netherlands; (1) orcid.org/0000-0001-5525-8322; Email: jasper.vandergucht@wur.nl

Wiebe M. de Vos - Membrane Science and Technology, MESA+ Institute for Nanotechnology, Faculty of Science and Technology, University of Twente, 7500 AE Enschede, The Netherlands; (1) orcid.org/0000-0002-0133-1931;

Email: w.m.devos@utwente.nl

\section{Authors}

Jiaying Li - Membrane Science and Technology, MESA+ Institute for Nanotechnology, Faculty of Science and Technology, University of Twente, 7500 AE Enschede, The Netherlands; $\odot$ orcid.org/0000-0002-1095-9771

Gerard van Ewijk - Akzo Nobel Decorative Coatings B.V., 2171 AJ Sassenheim, The Netherlands

Derk Jan van Dijken - BASF Nederland B.V., 8447 SN Heerenveen, The Netherlands

Complete contact information is available at: https://pubs.acs.org/10.1021/acsami.1c05031 


\section{Funding}

This work is a part of Advanced Research Center for Chemical Building Blocks, ARC-CBBC, which is co-founded and cofinanced by the Dutch Research Council (NWO) and the Netherlands Ministry of Economic Affairs and Climate.

\section{Notes}

The authors declare no competing financial interest.

\section{ACKNOWLEDGMENTS}

This work was supported by ARC-CBBC. J.v.d.G. acknowledges support from the European Research Council (ERC CoG Softbreak). The authors acknowledge Denys Pavlenko, Oguz Karvan, and Alberto Tena Matias for their help with gas permeation tests.

\section{REFERENCES}

(1) Lange, J.; Wyser, Y. Recent Innovations in Barrier Technologies for Plastic Packaging-a Review. Packag. Technol. Sci. 2003, 16, 149158.

(2) Vasile, C. Polymeric Nanocomposites and Nanocoatings for Food Packaging: A Review. Materials 2018, 11, No. 1834.

(3) Roilo, D.; Maestri, C. A.; Scarpa, M.; Bettotti, P.; Checchetto, R. Gas Barrier and Optical Properties of Cellulose Nanofiber Coatings with Dispersed $\mathrm{TiO}_{2}$ Nanoparticles. Surf. Coat. Technol. 2018, 343, 131-137.

(4) Abbas, M.; Buntinx, M.; Deferme, W.; Peeters, R. (Bio)Polymer/ Zno Nanocomposites for Packaging Applications: A Review of Gas Barrier and Mechanical Properties. Nanomaterials 2019, 9, No. 1494.

(5) Yu, J.; Ruengkajorn, K.; Crivoi, D.-G.; Chen, C.; Buffet, J.-C.; O'Hare, D. High Gas Barrier Coating Using Non-Toxic Nanosheet Dispersions for Flexible Food Packaging Film. Nat. Commun. 2019, 10, No. 2398.

(6) Li, F.; Zhang, C.; Weng, Y.; Diao, X.; Zhou, Y.; Song, X. Enhancement of Gas Barrier Properties of Graphene Oxide/Poly (Lactic Acid) Films Using a Solvent-Free Method. Materials 2020, 13, No. 3024.

(7) Bumbudsanpharoke, N.; Ko, S. Nanoclays in Food and Beverage Packaging. J. Nanomater. 2019, 2019, No. 8927167.

(8) Jung, J.; Deng, Z.; Zhao, Y. A Review of Cellulose Nanomaterials Incorporated Fruit Coatings with Improved Barrier Property and Stability: Principles and Applications. J. Food Process Eng. 2020, 43, No. e13344.

(9) Zabihzadeh Khajavi, M.; Ebrahimi, A.; Yousefi, M.; Ahmadi, S.; Farhoodi, M.; Mirza Alizadeh, A.; Taslikh, M. Strategies for Producing Improved Oxygen Barrier Materials Appropriate for the Food Packaging Sector. Food Eng. Rev. 2020, 12, 346-363.

(10) Meka, V. S.; Sing, M. K. G.; Pichika, M. R.; Nali, S. R.; Kolapalli, V. R. M.; Kesharwani, P. A Comprehensive Review on Polyelectrolyte Complexes. Drug Discovery Today 2017, 22, 16971706.

(11) Decher, G.; Hong, J. D.; Schmitt, J. Buildup of Ultrathin Multilayer Films by a Self-Assembly Process: Iii. Consecutively Alternating Adsorption of Anionic and Cationic Polyelectrolytes on Charged Surfaces. Thin Solid Films 1992, 210-211, 831-835.

(12) Priolo, M. A.; Holder, K. M.; Guin, T.; Grunlan, J. C. Recent Advances in Gas Barrier Thin Films Via Layer-by-Layer Assembly of Polymers and Platelets. Macromol. Rapid Commun. 2015, 36, 866879.

(13) Chen, J.-T.; Fu, Y.-J.; An, Q.-F.; Lo, S.-C.; Huang, S.-H.; Hung, W.-S.; Hu, C.-C.; Lee, K.-R.; Lai, J.-Y. Tuning Nanostructure of Graphene Oxide/Polyelectrolyte Lbl Assemblies by Controlling Ph of Go Suspension to Fabricate Transparent and Super Gas Barrier Films. Nanoscale 2013, 5, 9081-9088.

(14) Zhao, Q.; An, Q.-F.; Liu, T.; Chen, J.-T.; Chen, F.; Lee, K.-R.; Gao, C.-J. Bio-Inspired Polyelectrolyte Complex/Graphene Oxide Nanocomposite Membranes with Enhanced Tensile Strength and Ultra-Low Gas Permeability. Polym. Chem. 2013, 4, 4298-4302.
(15) Yang, Y.-H.; Haile, M.; Park, Y. T.; Malek, F. A.; Grunlan, J. C. Super Gas Barrier of All-Polymer Multilayer Thin Films. Macromolecules 2011, 44, 1450-1459.

(16) Yang, Y.-H.; Bolling, L.; Haile, M.; Grunlan, J. C. Improving Oxygen Barrier and Reducing Moisture Sensitivity of Weak Polyelectrolyte Multilayer Thin Films with Crosslinking. RSC Adv. 2012, 2, 12355-12363.

(17) Song, Y.; Meyers, K. P.; Gerringer, J.; Ramakrishnan, R. K.; Humood, M.; Qin, S.; Polycarpou, A. A.; Nazarenko, S.; Grunlan, J. C. Fast Self-Healing of Polyelectrolyte Multilayer Nanocoating and Restoration of Super Oxygen Barrier. Macromol. Rapid Commun. 2017, 38, No. 1700064.

(18) Kim, T.; Tran, T. H.; Hwang, S. Y.; Park, J.; Oh, D. X.; Kim, B.S. Crab-on-a-Tree: All Biorenewable, Optical and Radio Frequency Transparent Barrier Nanocoating for Food Packaging. ACS Nano 2019, 13, 3796-3805.

(19) Haile, M.; Sarwar, O.; Henderson, R.; Smith, R.; Grunlan, J. C. Polyelectrolyte Coacervates Deposited as High Gas Barrier Thin Films. Macromol. Rapid Commun. 2017, 38, No. 1600594.

(20) Smith, R. J.; Long, C. T.; Grunlan, J. C. Transparent Polyelectrolyte Complex Thin Films with Ultralow Oxygen Transmission Rate. Langmuir 2018, 34, 11086-11091.

(21) He, T.; Chan, V. Covalent Layer-by-Layer Assembly of Polyethyleneimine Multilayer for Antibacterial Applications. J. Biomed. Mater. Res., Part A 2010, 95A, 454-464.

(22) Shi, G. M.; Zuo, J.; Tang, S. H.; Wei, S.; Chung, T. S. Layer-byLayer (Lbl) Polyelectrolyte Membrane with Nexar Polymer as a Polyanion for Pervaporation Dehydration of Ethanol. Sep. Purif. Technol. 2015, 140, 13-22.

(23) Wang, Q.; Schlenoff, J. B. The Polyelectrolyte Complex/ Coacervate Continuum. Macromolecules 2014, 47, 3108-3116.

(24) Chollakup, R.; Beck, J. B.; Dirnberger, K.; Tirrell, M.; Eisenbach, C. D. Polyelectrolyte Molecular Weight and Salt Effects on the Phase Behavior and Coacervation of Aqueous Solutions of Poly(Acrylic Acid) Sodium Salt and Poly(Allylamine) Hydrochloride. Macromolecules 2013, 46, 2376-2390.

(25) Gratson, G. M.; Lewis, J. A. Phase Behavior and Rheological Properties of Polyelectrolyte Inks for Direct-Write Assembly. Langmuir 2005, 21, 457-464.

(26) Chollakup, R.; Smitthipong, W.; Eisenbach, C. D.; Tirrell, M. Phase Behavior and Coacervation of Aqueous Poly(Acrylic Acid)Poly(Allylamine) Solutions. Macromolecules 2010, 43, 2518-2528.

(27) Xu, M.; Lewis, J. A. Phase Behavior and Rheological Properties of Polyamine-Rich Complexes for Direct-Write Assembly. Langmuir 2007, 23, 12752-12759.

(28) Kamp, J.; Emonds, S.; Borowec, J.; Restrepo Toro, M. A.; Wessling, M. On the Organic Solvent Free Preparation of Ultrafiltration and Nanofiltration Membranes Using Polyelectrolyte Complexation in an All Aqueous Phase Inversion Process. J. Membr. Sci. 2021, 618, No. 118632.

(29) Meng, S.; Liu, Y.; Yeo, J.; Ting, J. M.; Tirrell, M. V. Effect of Mixed Solvents on Polyelectrolyte Complexes with Salt. Colloid Polym. Sci. 2020, 298, 887-894.

(30) Sun, Y.; Peng, C.; Wang, X.; Wang, R.; Chen, Y.; Zhang, D. Phase Behavior of Polyelectrolyte Complexes and Rheological Behavior of Alumina Suspensions for Direct Ink Writing. J. Am. Ceram. Soc. 2016, 99, 1902-1910.

(31) Swift, T.; Swanson, L.; Geoghegan, M.; Rimmer, S. The PhResponsive Behaviour of Poly(Acrylic Acid) in Aqueous Solution Is Dependent on Molar Mass. Soft Matter 2016, 12, 2542-2549.

(32) Choi, J.; Rubner, M. F. Influence of the Degree of Ionization on Weak Polyelectrolyte Multilayer Assembly. Macromolecules 2005, 38, $116-124$.

(33) Demadis, K. D.; Paspalaki, M.; Theodorou, J. Controlled Release of Bis(Phosphonate) Pharmaceuticals from Cationic Biodegradable Polymeric Matrices. Ind. Eng. Chem. Res. 2011, 50, 58735876 . 
(34) Kong, H. J.; Mooney, D. J. The Effects of Poly(Ethyleneimine)

(Pei) Molecular Weight on Reinforcement of Alginate Hydrogels. Cell Transplant. 2003, 12, 779-785.

(35) Tomar, B. S.; Shahin, A.; Tirumkudulu, M. S. Cracking in Drying Films of Polymer Solutions. Soft Matter 2020, 16, 3476-3484.

(36) Hoerter, M.; Oprea, A.; Bârsan, N.; Weimar, U. Chemical Interaction of Gaseous Ammonia and Water Vapour with Polyacrylic Acid Layers. Sens. Actuators, B 2008, 134, 743-749.

(37) Kirwan, L. J.; Fawell, P. D.; van Bronswijk, W. In Situ Ftir-Atr Examination of Poly(Acrylic Acid) Adsorbed onto Hematite at Low $\mathrm{Ph}$. Langmuir 2003, 19, 5802-5807.

(38) Kasprzak, A.; Popławska, M.; Bystrzejewski, M.; Łabędź, O.; Grudziński, I. P. Conjugation of Polyethylenimine and Its Derivatives to Carbon-Encapsulated Iron Nanoparticles. RSC Adv. 2015, 5, $85556-85567$.

(39) Müller, M. The Anomalous Influence of Polyelectrolyte Concentration on the Deposition and Nanostructure of Poly(Ethyleneimine)/Poly(Acrylic Acid) Multilayers. Molecules 2019, 24, No. 2141.

(40) Gu, X.; Raghavan, D.; Douglas, J. F.; Karim, A. Hole-Growth Instability in the Dewetting of Evaporating Polymer Solution Films. J. Polym. Sci., Part B: Polym. Phys. 2002, 40, 2825-2832.

(41) Mendelsohn, J. D.; Barrett, C. J.; Chan, V. V.; Pal, A. J.; Mayes, A. M.; Rubner, M. F. Fabrication of Microporous Thin Films from Polyelectrolyte Multilayers. Langmuir 2000, 16, 5017-5023.

(42) Mohammed, M. A.; Sproncken, C. C. M.; Gumí-Audenis, B.; Lazdanaité, E.; Stabile, R.; Voets, I. K.; Raz, O. Reversibly Programmable Photonics Via Responsive Polyelectrolyte Multilayer Cladding. Adv. Opt. Mater. 2020, 8, No. 2000325.

(43) Hiller, J. A.; Mendelsohn, J. D.; Rubner, M. F. Reversibly Erasable Nanoporous Anti-Reflection Coatings from Polyelectrolyte Multilayers. Nat. Mater. 2002, 1, 59-63.

(44) Baker, R. W. Membrane Transport Theory. Membrane Technology and Applications; John Wiley \& Sons, Inc., 2012; pp 15-96.

(45) Song, Y.; Tzeng, P.; Grunlan, J. C. Super Oxygen and Improved Water Vapor Barrier of Polypropylene Film with Polyelectrolyte Multilayer Nanocoatings. Macromol. Rapid Commun. 2016, 37, 963968.

(46) Schmid, M.; Dallmann, K.; Bugnicourt, E.; Cordoni, D.; Wild, F.; Lazzeri, A.; Noller, K. Properties of Whey-Protein-Coated Films and Laminates as Novel Recyclable Food Packaging Materials with Excellent Barrier Properties. Int. J. Polym. Sci. 2012, 2012, No. 562381. 